
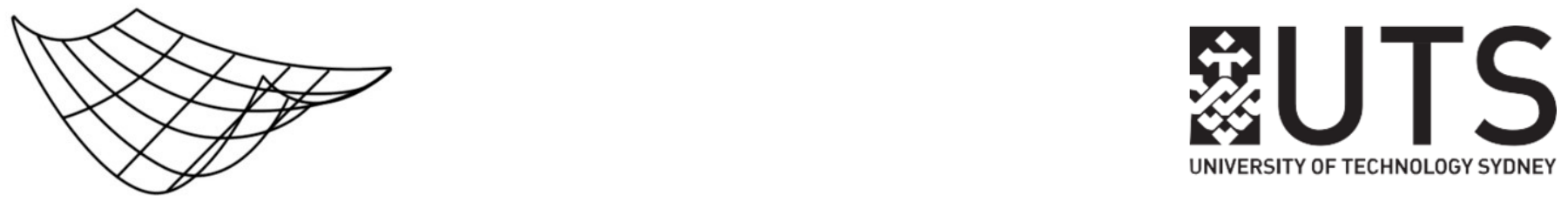

QUANTITATIVE FINANCE RESEARCH
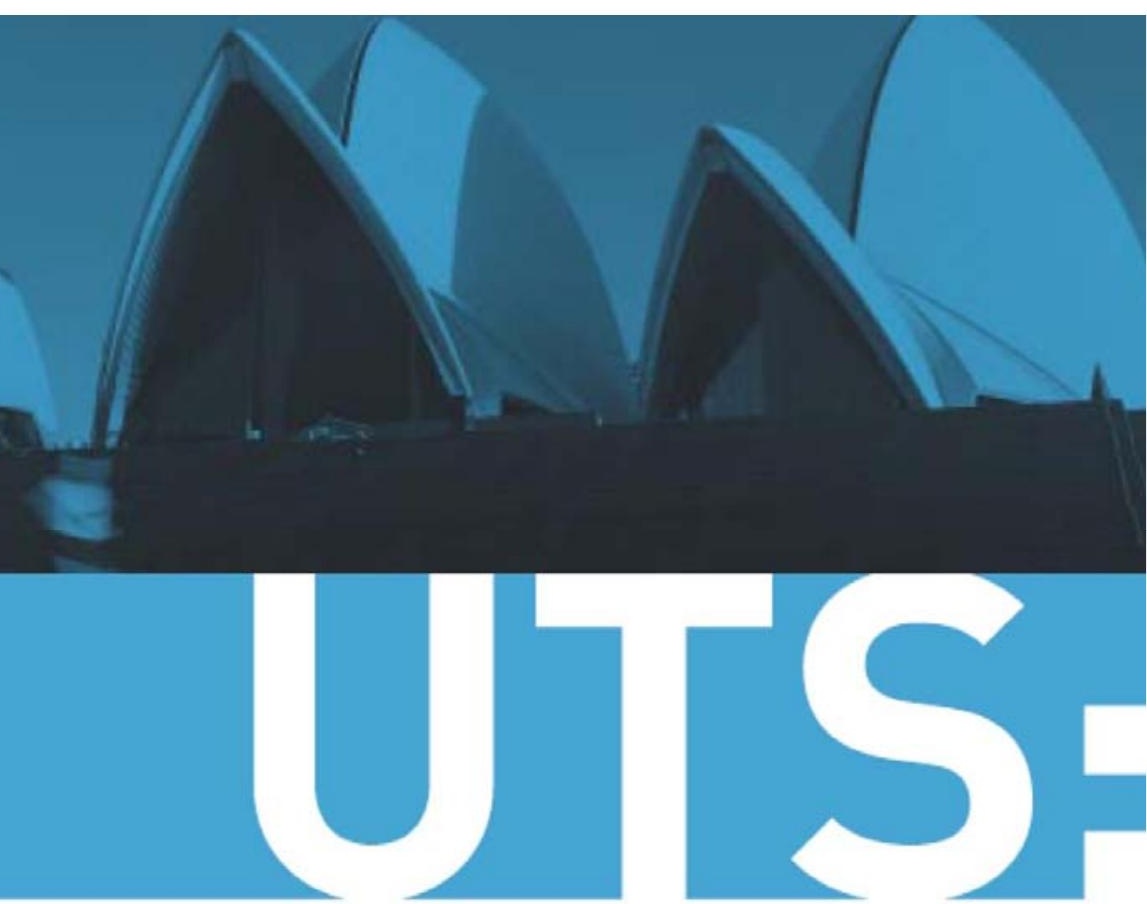

THINK.CHANGE.DO

\title{
QUANTITATIVE FINANCE RESEARCH
}

\section{Sure Profits via Flash Strategies and the Impossibility of Predictable Jumps}

Claudio Fontana, Markus Pelger, And Eckhard Platen 


\title{
SURE PROFITS VIA FLASH STRATEGIES AND THE IMPOSSIBILITY OF PREDICTABLE JUMPS
}

\author{
CLAUDIO FONTANA, MARKUS PELGER, AND ECKHARD PLATEN
}

\begin{abstract}
In an arbitrage-free financial market, asset prices should not exhibit jumps of a predictable magnitude at predictable times. We provide a rigorous formulation of this result in a fully general setting, only allowing for buy-and-hold positions and without imposing any semimartingale restriction. We show that asset prices do not exhibit predictable jumps if and only if there is no possibility of obtaining sure profits via high-frequency limits of buy-and-hold trading strategies. Our results imply that, under minimal assumptions, price changes occurring at scheduled dates should only be due to unanticipated information releases.
\end{abstract}

\section{INTRODUCTION}

In the financial markets literature, the importance of allowing for jumps in asset prices at scheduled or predictable dates is widely acknowledged. Indeed, asset prices typically change in correspondence of macroeconomic news announcements (see [Eva11, KW14, KV91, LM08, Ran11]), publication of earnings reports (see [DJ05, LM08]), dividend payments (see [HJ88]), Federal Reserve meetings (see [Pia01, Pia05]), major political decisions 1] and all these events take place at dates which are known in advance. In the context of continuous-time models, Lee12] reports significant empirical evidence on jump predictability, while a model of the US Treasury rate term structure with jumps occurring in correspondence of employment report announcement dates is developed in [KW14] (see also GS16] in the case of credit risky term structures). Hence, realistic financial models should account for the presence of jumps arriving at predictable times.

According to the efficient market hypothesis, asset prices should fully reflect all available information (see [Fam70]). In particular, if asset prices suddenly change at scheduled or predictable dates, then this can only be due to the release of unanticipated information. Indeed, under market efficiency, if the information released does not contain any surprise element, then it should be already incorporated in asset prices and, hence, prices should not move. This implication of market efficiency is also coherent with absence of arbitrage: if a price process is known to jump at a given

Date: August 2, 2017.

2010 Mathematics Subject Classification. 60G07, 60G44, 91G99.

Key words and phrases. Absence of arbitrage; predictable time; semimartingale; high-frequency trading.

JEL Classification. C02, G12, G14.

The first author gratefully acknowledges the support of the Bruti-Liberati Visiting Fellowship and the hospitality of the Quantitative Finance Research Centre at the Finance Discipline Group at University of Technology Sydney.

${ }^{1}$ Recent political events like the Brexit and the election of the American president in 2016 represent striking examples of the impact on financial markets of discontinuities happening at scheduled dates. 
point in time, then the size of the jump should not be perfectly known in advance, otherwise arbitrage profits would be possible. As pointed out in [ABD10, Sect. 2.1], this can be easily understood by analogy to discrete-time models, where absence of arbitrage implies that the return over each single trading period can never be known in advance. Summing up, market efficiency and absence of arbitrage suggest that asset prices cannot exhibit predictable jumps, i.e., discontinuities such that the time of the jump and the size of the jump can be known in advance.

The goal of the present paper is to characterize the minimal no-arbitrage condition under which asset prices do not exhibit predictable jumps. We restrict from making any assumption on the price process, except for mild path regularity. In particular, we do not assume the semimartingale property. We only allow for realistic trading strategies, consisting of bounded buy-and-hold positions and high-frequency limits thereof, which we name flash strategies (see Definition 2.2). Our main result (Theorem 2.3) shows that the existence of predictable jumps is equivalent to the possibility of realizing sure profits via flash strategies. Moreover, in the semimartingale case, those sure profits can be even realized instantaneously (see Corollary 2.5). In view of these results, we argue that any reasonable financial model should not allow for sure profits via flash strategies and, therefore, the time of a jump and/or the size of the jump should always be unpredictable.

Our results are closely related to arbitrage possibilities in high-frequency markets. In particular, our notion of flash strategy is similar to a directional event-based strategy (see e.g. [Ald13, Chap. 9]). Such strategies aim at realizing positive profits in correspondence of some predetermined market events. In the case of anticipated events, such as scheduled macroeconomic announcements, the strategy is opened ahead of the event and liquidated just after the event. The holding period is typically very short and the speed of response determines the trade gain. Our notion of flash strategy can also represent a latency arbitrage strategy (see e.g. [Ald13, Chap. 12]): if the same asset is traded in two markets at slightly different prices, then high-frequency traders can arbitrage the price difference by simultaneously trading in the two markets. Since our price process is allowed to be vector-valued, this situation can be easily captured by representing the prices of the same asset on different markets as different components of the vector price process. Other kinds of high-frequency strategies that can be represented via flash strategies include front-running strategies, as described in the recent best-selling book [Lew14]. Our results indicate that the existence of predictable jumps lies at the origin of sure profits generated by all these types of high-frequency strategies.

The existence of sure profits generated by predictable jumps is also related to the classical issue of the behavior of ex-dividend prices at dividend payment dates, as considered in [HJ88] (see also [Bat03]). Typically, the dividend payment date and the amount of the dividend are known in advance (i.e., they are predictable). [HJ88] show that, if there exists a martingale measure, then either the ex-dividend price drops exactly by the amount of the dividend or the jump in the exdividend price cannot be predictable. In this perspective, our results can be regarded as the most general formulation of the classical result of [HJ88] (to this effect, see also Remark 2.4).

The rest of the paper is organized as follows. In Section 2.1 we introduce the probabilistic setting, and in Section 2.2 we define the class of trading strategies under consideration. Section 2.3 contains 
our main result, which is specialized in the semimartingale case in Section 2.4. We briefly discuss in Section 2.5 the relations with other no-arbitrage conditions and we then conclude in Section 3.

\section{Characterizing PREDiCTABle JUmps in terms of SURE PROFits VIA Flash Strategies}

2.1. Setting. Let $(\Omega, \mathcal{F}, \mathbb{P})$ be a probability space endowed with a filtration $\mathbb{F}=\left(\mathcal{F}_{t}\right)_{t \geq 0}$ satisfying the usual conditions of right-continuity and completeness and supporting a càdlàg (right-continuous with left limits) real-valued ${ }^{2}$ adapted process $X=\left(X_{t}\right)_{t \geq 0}$. The filtration $\mathbb{F}$ represents the flow of available information, while the process $X$ represents the gains process of a risky asset, discounted with respect to some baseline security. In the case of a dividend paying asset, this corresponds to the sum of the discounted ex-dividend price and the cumulated discounted dividends. We do not assume that $X$ is a semimartingale nor that the initial $\sigma$-field $\mathcal{F}_{0}$ is trivial. The results presented below apply to any model in a finite horizon $T<+\infty$ by simply considering the stopped process $X^{T}$. We refer to [JS03] for all unexplained notions related to the general theory of stochastic processes.

Since $X$ is càdlàg and adapted, there exists a sequence of stopping times $\left(T_{n}\right)_{n \in \mathbb{N}}$ exhausting the jumps of $X$, in the sense that $\{\Delta X \neq 0\}=\bigcup_{n \in \mathbb{N}} \llbracket T_{n} \rrbracket$ (see [JS03, Proposition I.1.32]) $)^{3}$. We say that exhibits predictable jumps if there exists at least one jump time $T_{n}$ which is a predictable time and such that the random variable $\Delta X_{T_{n}} \mathbf{1}_{\left\{T_{n}<+\infty\right\}}$ is $\mathcal{F}_{T_{n}-}$-measurable. In other words, $X$ exhibits predictable jumps if there exists at least one predictable jump time at which the size of the jump is known just before the occurrence of the jump. We aim at characterizing the absence of predictable jumps in terms of a minimal and realistic no-arbitrage property.

2.2. Trading strategies. We describe the activity of trading in the financial market according to the following definition.

Definition 2.1. A buy-and-hold strategy is a stochastic process $h$ of the form $h=\xi \mathbf{1}_{\rrbracket \sigma}, \tau$, where $\sigma$ and $\tau$ are two bounded stopping times such that $\sigma \leq \tau$ a.s. and $\xi$ is a bounded $\mathcal{F}_{\sigma}$-measurable random variable.

A buy-and-hold strategy corresponds to the simplest possible trading strategy, where a portfolio $\xi$ is created at time $\sigma$ and liquidated at time $\tau$. Note that the portfolio $\xi$ is restricted to be bounded, thus excluding the case of arbitrarily large positions in the available assets. For a buy-and-hold strategy $h$, the gains from trading at date $t$ are given by $(h \cdot X)_{t}:=\xi\left(X_{\tau \wedge t}-X_{\sigma \wedge t}\right)$, for $t \geq 0$.

Definition 2.2. A flash strategy is a sequence $\left(h^{n}\right)_{n \in \mathbb{N}}$ of buy-and-hold strategies $h^{n}=\xi^{n} \mathbf{1}_{\rrbracket \sigma_{n}, \tau_{n} \rrbracket}$ such that the random variables $\left(\xi^{n}\right)_{n \in \mathbb{N}}$ are bounded uniformly in $n$ and the following two properties hold a.s. for $n \rightarrow+\infty$ :

(i) the sequences $\left(\sigma_{n}\right)_{n \in \mathbb{N}}$ and $\left(\tau_{n}\right)_{n \in \mathbb{N}}$ converge to some stopping time $\tau$ with $\mathbb{P}(\tau<+\infty)>0$;

(ii) the random variables $\left(\xi^{n}\right)_{n \in \mathbb{N}}$ converge to some random variable $\xi$.

\footnotetext{
${ }^{2}$ We restrict our presentation to the case of a one-dimensional process $X$ for clarity of notation only. The multidimensional case is completely analogous and can be treated with the same tools.

${ }^{3}$ We recall that the graph of a stopping time $T_{n}$ is defined as $\llbracket T_{n} \rrbracket=\left\{(\omega, t) \in \Omega \times \mathbb{R}_{+}: T_{n}(\omega)=t\right\}$. Similarly, for two stopping times $\sigma$ and $\tau$, we define the stochastic interval $\rrbracket \sigma, \tau \rrbracket=\left\{(\omega, t) \in \Omega \times \mathbb{R}_{+}: \sigma(\omega)<t \leq \tau(\omega)\right\}$.
} 
A flash strategy $\left(h^{n}\right)_{n \in \mathbb{N}}$ is said to generate a sure profit if $\left(h^{n} \cdot X\right)_{t}$ converges a.s. to $c \mathbf{1}_{\{\tau \leq t\}}$, for all $t \geq 0$, for some constant $c>0$.

A flash strategy represents the possibility of investing at higher and higher frequencies. In the limit case, the strategy converges to a bounded position $\xi$ which is constructed and then immediately liquidated at some random time $\tau$. If by doing so and starting from zero initial wealth an investor can reach a strictly positive deterministic amount of wealth (provided that the investor trades at all, i.e., from time $\tau$ onwards), then the flash strategy is said to generate a sure profit. The requirement that the positions $\left(\xi^{n}\right)_{n \in \mathbb{N}}$ are uniformly bounded means that an investor is not allowed to make larger and larger trades as the holding period $\tau_{n}-\sigma_{n}$ converges to zero. Note also that a sure profit does not involve any risk, since in the limit the gains from trading are uniformly bounded from below by zero. Clearly, if a flash strategy generates a sure profit for some $c>0$, then there exist sure profits for every $c>0$, since the strategy can be rescaled to arbitrarily large values.

2.3. The main result. The following theorem shows that the absence of predictable jumps is equivalent to the absence of sure profits via flash strategies.

Theorem 2.3. The process $X$ does not exhibit predictable jumps if and only if there are no sure profits via flash strategies.

Proof. We first prove that if $X$ exhibits predictable jumps, then there exist sure profits. To this effect, let $T$ be a predictable time with $\llbracket T \rrbracket \subseteq\{\Delta X \neq 0\}$ such that the random variable

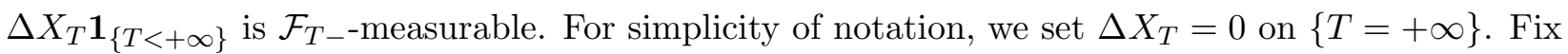
some constant $k \geq 1$ such that $\mathbb{P}\left(T<+\infty,\left|\Delta X_{T}\right| \in[1 / k, k]\right)>0$ and define the stopping time $\tau:=T \mathbf{1}_{\left\{\left|\Delta X_{T}\right| \in[1 / k, k]\right\}}+\infty \mathbf{1}_{\left\{\left|\Delta X_{T}\right| \notin[1 / k, k]\right\}}$. By [JS03, Proposition I.2.10], $\tau$ is a predictable time and, therefore, in view of [JS03, Theorem I.2.15], there exists an announcing sequence $\left(\rho_{n}\right)_{n \in \mathbb{N}}$ of stopping times satisfying $\rho_{n}<\tau$ and such that $\rho_{n}$ increases to $\tau$ for $n \rightarrow+\infty$. For each $n \in \mathbb{N}$, let $\sigma_{n}:=\rho_{n} \wedge n$ and $\tau_{n}:=\tau \wedge n$ and define the sequence $\left(h^{n}\right)_{n \in \mathbb{N}}$ by

$$
h^{n}=\xi^{n} \mathbf{1}_{\left.\rrbracket \sigma_{n}, \tau_{n}\right]}, \quad \text { where } \xi^{n}:=\frac{\mathbf{1}_{\left\{\left|\mathbb{E}\left[\Delta X_{\tau} \mid \mathcal{F}_{\sigma_{n}}\right]\right| \in[1 / k, k]\right\}}}{\mathbb{E}\left[\Delta X_{\tau} \mid \mathcal{F}_{\sigma_{n}}\right]}, \quad \text { for every } n \in \mathbb{N},
$$

with the convention $\Delta X_{\tau}=0$ on $\{\tau=+\infty\}$. By construction, it holds that $\left|\xi^{n}\right| \leq k$, for every $n \in \mathbb{N}$, so that $\left(h^{n}\right)_{n \in \mathbb{N}}$ is well-defined as a sequence of buy-and-hold strategies. Moreover, as a consequence of the martingale convergence theorem, the random variables $\left(\xi^{n}\right)_{n \in \mathbb{N}}$ converge a.s. to

$$
\xi:=\frac{\mathbf{1}_{\left\{\left|\mathbb{E}\left[\Delta X_{\tau} \mid \mathcal{F}_{\tau-}\right]\right| \in[1 / k, k]\right\}}}{\mathbb{E}\left[\Delta X_{\tau} \mid \mathcal{F}_{\tau-}\right]}=\frac{\mathbf{1}_{\left\{\left|\Delta X_{\tau}\right| \in[1 / k, k]\right\}}}{\Delta X_{\tau}}=\frac{\mathbf{1}_{\left\{\Delta X_{\tau} \neq 0\right\}}}{\Delta X_{\tau}},
$$

where the second equality makes use of the fact that $\Delta X_{\tau}$ is $\mathcal{F}_{\tau-}$-measurable, as follows from the

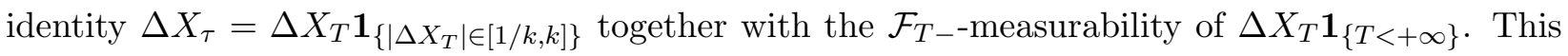
shows that $\left(h^{n}\right)_{n \in \mathbb{N}}$ is a flash strategy in the sense of Definition 2.2. To prove that it generates a sure profit, it suffices to remark that, for every $t \geq 0$, it holds that $\lim _{n \rightarrow+\infty} X_{\tau_{n} \wedge t}=X_{\tau \wedge t}$ and

$$
\lim _{n \rightarrow+\infty} X_{\sigma_{n} \wedge t}=X_{\tau-} \mathbf{1}_{\{\tau \leq t\}}+X_{t} \mathbf{1}_{\{\tau>t\}}
$$


so that

$$
\lim _{n \rightarrow+\infty}\left(h^{n} \cdot X\right)_{t}=\lim _{n \rightarrow+\infty}\left(\xi^{n}\left(X_{\tau_{n} \wedge t}-X_{\sigma_{n} \wedge t}\right)\right)=\xi \Delta X_{\tau} \mathbf{1}_{\{\tau \leq t\}}=\mathbf{1}_{\{\tau \leq t\}} \quad \text { a.s. }
$$

This shows that $\left(h^{n}\right)_{n \in \mathbb{N}}$ generates a sure profit with respect to the constant $c=1$.

We now turn to the converse implication. Let $\left(h^{n}\right)_{n \in \mathbb{N}}$ be a flash strategy, composed by elements of the form $h^{n}=\xi^{n} \mathbf{1}_{\rrbracket \sigma_{n}, \tau_{n} \rrbracket}$, generating a sure profit with respect to $c>0$ and a stopping time $\tau$. It can be checked that $\lim _{k \rightarrow+\infty}\left(h^{n} \cdot X\right)_{t-1 / k}=\left(h^{n} \cdot X\right)_{t-}$ uniformly over $n \in \mathbb{N}$. Indeed, defining $\bar{\xi}:=\sup _{n \in \mathbb{N}}\left|\xi^{n}\right|$ (which is a bounded random variable due to Definition 2.2), it holds that

$$
\begin{aligned}
& \lim _{k \rightarrow+\infty} \sup _{n \in \mathbb{N}}\left|\left(h^{n} \cdot X\right)_{t-\frac{1}{k}}-\left(h^{n} \cdot X\right)_{t-}\right|=\lim _{k \rightarrow+\infty} \sup _{n \in \mathbb{N}}\left|\xi^{n}\left(X_{t-\frac{1}{k}}^{\tau_{n}}-X_{t-\frac{1}{k}}^{\sigma_{n}}\right)-\xi^{n}\left(X_{t-}^{\tau_{n}}-X_{t-}^{\sigma_{n}}\right)\right| \\
& \quad \leq \bar{\xi} \lim _{k \rightarrow+\infty} \sup _{n \in \mathbb{N}}\left|-\mathbf{1}_{\left\{t-\frac{1}{k} \leq \tau_{n}<t\right\}}\left(X_{\tau_{n}}-X_{\sigma_{n}}\right)+\mathbf{1}_{\left\{\sigma_{n}<t-\frac{1}{k} \leq \tau_{n}\right\}}\left(X_{t-\frac{1}{k}}-X_{\sigma_{n}}\right)-\mathbf{1}_{\left\{\sigma_{n}<t \leq \tau_{n}\right\}}\left(X_{t-}-X_{\sigma_{n}}\right)\right| \\
& \quad \leq \bar{\xi} \lim _{k \rightarrow+\infty}\left(\sup _{n \in \mathbb{N}} \mathbf{1}_{\left\{t-\frac{1}{k} \leq \tau_{n}<t\right\}}\left|X_{\tau_{n}}-X_{t-}\right|+\sup _{n \in \mathbb{N}} \mathbf{1}_{\left\{t-\frac{1}{k} \leq \sigma_{n}<t\right\}}\left|X_{\sigma_{n}}-X_{t-}\right|\right) \\
& \quad \leq 2 \bar{\xi} \lim _{k \rightarrow+\infty} \sup _{u \in\left[t-\frac{1}{k}, t\right)}\left|X_{u}-X_{t-}\right|=0 .
\end{aligned}
$$

By the Moore-Osgood theorem, we can then conclude that, for every $t \geq 0$,

$$
c \mathbf{1}_{\{t=\tau\}}=\lim _{n \rightarrow+\infty}\left(h^{n} \cdot X\right)_{t}-\lim _{k \rightarrow+\infty} \lim _{n \rightarrow+\infty}\left(h^{n} \cdot X\right)_{t-\frac{1}{k}}=\Delta X_{t} \lim _{n \rightarrow+\infty} h_{t}^{n} \quad \text { a.s. }
$$

Letting $\xi=\lim _{n \rightarrow+\infty} \xi^{n}$ (see Definition 2.2), a first implication of 2.1) is that $\{\tau<+\infty\} \subseteq\{\xi \neq 0\}$ and $\llbracket \tau \rrbracket \subseteq\{\Delta X \neq 0\}$, up to an evanescent set, so that $\tau$ is a jump time of $X$. Furthermore, always by (2.1), it holds that $\Delta X_{\tau}=c /\left(\lim _{n \rightarrow+\infty} \xi^{n} \mathbf{1}_{\left\{\sigma_{n}<\tau \leq \tau_{n}\right\}}\right)$ a.s. on $\{\tau<+\infty\}$. Noting that the random

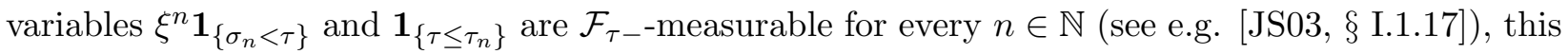

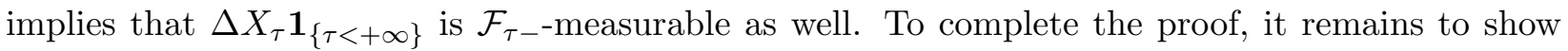
that $\tau$ is a predictable time. For each $n \in \mathbb{N}$, let $A_{n}:=\left\{\sigma_{n}<\tau \leq \tau_{n}\right\} \cap\left\{\xi^{n} \neq 0\right\}$ and note that $A_{n} \subseteq\{\tau<+\infty\}$, since each stopping time $\tau_{n}$ is bounded. Moreover, it holds that

$$
\lim _{n \rightarrow+\infty} \mathbf{1}_{A_{n}}=\lim _{n \rightarrow+\infty} \mathbf{1}_{\left\{\sigma_{n}<\tau \leq \tau_{n}\right\}} \xi^{n} \frac{\mathbf{1}_{\left\{\xi^{n} \neq 0\right\}}}{\xi^{n}}=\frac{c}{\xi \Delta X_{\tau}} \mathbf{1}_{\{\tau<+\infty\}} \quad \text { a.s. }
$$

This identity shows that the sequence $\left(A_{n}\right)_{n \in \mathbb{N}}$ is convergent, with $\lim _{n \rightarrow+\infty} A_{n}=\{\tau<+\infty\}$ and $\xi \Delta X_{\tau}=c$ on $\{\tau<+\infty\}$ (up to a $\mathbb{P}$-nullset). Since the stopping times $\left(\sigma_{n}\right)_{n \in \mathbb{N}}$ and $\left(\tau_{n}\right)_{n \in \mathbb{N}}$ converge

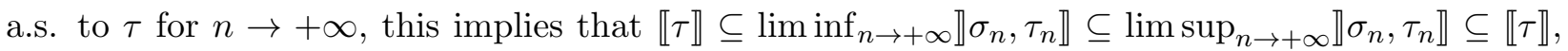
so that $\llbracket \tau \rrbracket=\lim _{n \rightarrow+\infty} \rrbracket \sigma_{n}, \tau_{n} \rrbracket$. Since each stochastic interval $\rrbracket \sigma_{n}, \tau_{n} \rrbracket$ is a predictable set (see e.g. [JS03, Proposition I.2.5]), it follows that $\llbracket \tau \rrbracket$ is also a predictable set, i.e., $\tau$ is a predictable time.

Remark 2.4. Classical examples of models allowing for sure profits via flash strategies are given by the escrowed dividend models introduced in [Rol77, Ges79, Wha81]. Indeed, such models consider an asset paying a deterministic dividend at a known date and assume that the ex-dividend price drops by a fixed fraction of the dividend at the dividend payment date (see also [HJ88]). This corresponds to a predictable jump of the process $X$ and, hence, in view of Theorem 2.3 , can be exploited to generate a sure profit via a flash strategy. 
2.4. The semimartingale case. Theorem 2.3 holds true for any càdlàg adapted process $X$. If in addition $X$ is assumed to be a semimartingale, then the absence of predictable jumps admits a further simple characterization. For a semimartingale $X$, we say that a bounded predictable process $h$ is an instantaneous strategy if it is of the form $h=\xi \mathbf{1}_{\llbracket \tau \rrbracket}$, for some bounded random variable $\xi$ and a stopping time $\tau$. In the spirit of Definition 2.2, we say that an instantaneous strategy $h$ generates sure profits if $(h \cdot X)_{t}=c \mathbf{1}_{\{\tau \leq t\}}$ a.s., for every $t \geq 0$, for some constant $c>0$.

Corollary 2.5. Assume that the process $X$ is a semimartingale. Then the following are equivalent:

(i) $X$ does not exhibit predictable jumps;

(ii) there are no sure profits via flash strategies;

(iii) there are no sure profits via instantaneous strategies.

Proof. $(i) \Rightarrow(i i)$ : this implication follows from Theorem 2.3. (ii) $\Rightarrow($ iii $)$ : let $\left(h^{n}\right)_{n \in \mathbb{N}}$ be a flash strategy, composed by elements $h^{n}=\xi^{n} \mathbf{1}_{\rrbracket \sigma_{n}, \tau_{n} \rrbracket}$, such that $\lim _{n \rightarrow+\infty}\left(h^{n} \cdot X\right)_{t}=c \mathbf{1}_{\{\tau \leq t\}}$ a.s., for every $t \geq 0$, for some $c>0$ and a stopping time $\tau$. As shown in the second part of the proof of Theorem $2.3 h^{n}$ converges a.s. to $h:=\xi \mathbf{1}_{\llbracket \tau \rrbracket}$ for $n \rightarrow+\infty$. The dominated convergence theorem for stochastic integrals (see [Pro04, Theorem IV.32]) then implies that $(h \cdot X)_{t}=\lim _{n \rightarrow+\infty}\left(h^{n} \cdot X\right)_{t}=c \mathbf{1}_{\{\tau \leq t\}}$ a.s., for every $t \geq 0$. (iii) $\Rightarrow(i)$ : let $h=\xi \mathbf{1}_{\llbracket \tau \rrbracket}$ be an instantaneous strategy generating sure profits. By [JS03, $\S$ I.4.38], it holds that $h \cdot X=\xi \Delta X_{\tau} \mathbf{1}_{\llbracket \tau,+\infty \llbracket}$, so that $\xi \Delta X_{\tau}=c$ a.s. on $\{\tau<+\infty\}$. This implies that $\tau$ is a jump time of $X$ and $\{\tau<+\infty\} \subseteq\{\xi \neq 0\}$. Moreover, the random variable $\Delta X_{\tau} \mathbf{1}_{\{\tau<+\infty\}}$ is $\mathcal{F}_{\tau-\text {-measurable, since }} \xi \mathbf{1}_{\{\tau<+\infty\}}$ is $\mathcal{F}_{\tau-\text {-measurable, due to the predictability of }}$ $h$. Finally, the predictability of $\tau$ follows by noting that $\llbracket \tau \rrbracket=\{h \neq 0\}$.

2.5. Comparison with other no-arbitrage conditions. In the semimartingale case, the absence of sure profits from instantaneous strategies must be regarded as a minimal no-arbitrage condition. In particular, it is implied by the requirement of no increasing profit (NIP), itself an extremely weak no-arbitrage condition for a semimartingale financial model (see [Fon15] and [KK07, Section 3.4])

The absence of predictable jumps can be directly proven by means of martingale methods under the classical no free lunch with vanishing risk (NFLVR) condition. Note, however, that NFLVR is much stronger than the absence of sure profits as considered above. We recall that NFLVR is equivalent to the existence of a probability measure $\mathbb{Q} \sim \mathbb{P}$ such that $X$ is a sigma-martingale under $\mathbb{Q}$ (see [DS98]). For completeness, we present the following proposition with its elementary proof.

Proposition 2.6. Assume that the process $X$ is a semimartingale satisfying NFLVR. Then $X$ cannot exhibit predictable jumps.

Proof. If $X$ satisfies NFLVR, then there exists $\mathbb{Q} \sim \mathbb{P}$ and an increasing sequence of predictable sets $\left(\Sigma_{k}\right)_{k \in \mathbb{N}}$ with $\bigcup_{k \in \mathbb{N}} \Sigma_{k}=\Omega \times \mathbb{R}_{+}$such that $\mathbf{1}_{\Sigma_{k}} \cdot X$ is a uniformly integrable martingale under $\mathbb{Q}$, for every $k \in \mathbb{N}$ (see [JS03, Definition III.6.33]). Let $T$ be a predictable time such that $\llbracket T \rrbracket \subseteq\{\Delta X \neq 0\}$

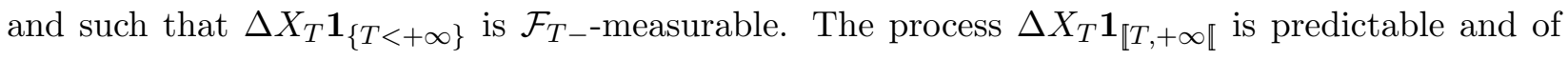

\footnotetext{
${ }^{4}$ An $X$-integrable predictable process $h$ is said to generate an increasing profit if the gains from trading process $h \cdot X$ is predictable, non-decreasing and satisfies $\mathbb{P}\left((h \cdot X)_{T}>0\right)>0$ for some $T>0$, see [Fon15, Definition 2.2].
} 
finite variation and

$$
Y^{(k)}:=\mathbf{1}_{\Sigma_{k}} \cdot\left(\Delta X_{T} \mathbf{1}_{\llbracket T,+\infty \llbracket}\right)=\mathbf{1}_{\Sigma_{k}} \cdot\left(\mathbf{1}_{\llbracket T \rrbracket} \cdot X\right)=\mathbf{1}_{\llbracket T \rrbracket} \cdot\left(\mathbf{1}_{\Sigma_{k}} \cdot X\right),
$$

for every $k \in \mathbb{N}$, where we have used [JS03, $\S I .4 .38]$ and the associativity of the stochastic integral. For every $k \in \mathbb{N}$, the process $Y^{(k)}$ is null (up to an evanescent set), being a predictable local martingale of finite variation (see [JS03, Corollary I.3.16]). In turn, this implies that $\Delta X_{T} \mathbf{1}_{\llbracket T,+\infty \llbracket}$ is null (up to an evanescent set), thus contradicting the assumption that $T$ is a jump time of $X$.

Remark 2.7. Predictable jumps can never occur under the no unbounded profit with bounded risk (NUPBR) condition, introduced in [KK07, Definition 4.1]. This follows by Proposition 2.6, noting that NUPBR is equivalent to NFLVR up to a localizing sequence of stopping times. In turn, since NUPBR is equivalent to the existence and finiteness of the growth-optimal portfolio (see [KK07, Theorem 4.12]), this implies that predictable jumps are excluded under the benchmark approach, see $[\mathrm{PH} 06]$.

\section{Conclusions}

In this paper we have shown that, under minimal assumptions, the existence of jumps of predictable magnitude occurring at predictable times is equivalent to the possibility of realizing sure profits via flash strategies (see Theorem 2.3). Since flash strategies represent well typical strategies adopted by high-frequency traders, as explained in the introduction, we deduce that the profitability of high-frequency strategies is closely related to the presence of information not yet incorporated in market prices. In this sense, the arbitrage activity of high-frequency traders has a beneficial role in price discovery and leads to an increase of market efficiency (see [BHR14] for empirical results in this direction). However, a general analysis of the impact of high-frequency trading is definitely beyond the scope of this short theoretical paper.

Finally, we want to emphasize that, since predictability depends on the reference filtration, the possibility of realizing sure profits via flash strategies depends on the information set under consideration. This means that financial markets can be efficient in the semi-strong form and sure profits via flash strategies impossible to achieve for ordinary investors having access to the publicly available information, while investors having access to privileged information (insider traders) can have an information set rich enough to allow for sure profits via flash strategies, so that market efficiency does not hold in the strong form. This is simply a consequence of Theorem 2.3 together with the fact that the predictable sigma-field associated to a smaller filtration is a subset of the predictable sigma-field associated to a larger filtration. This observation is in line with the empirical literature documenting violations to strong-form market efficiency in the presence of insider information (see e.g. [Fam91, Sect. 6]). This is also in line with the empirical analysis of [HLS15], where it is shown that institutional traders have an informational advantage which allows to predict to some extent the time and the content of news announcements as well as the returns on the announcement date. 


\section{REFERENCES}

[ABD10] T.G. Andersen, T. Bollerslev, and F.X. Diebold. Parametric and nonparametric volatility measurement. In Y. Aït-Sahalia and L.P. Hansen, editors, Handbook of Financial Econometrics, volume 1, chapter 2, pages 67-137. North-Holland, 2010.

[Ald13] I. Aldridge. High-Frequency Trading: A Practical Guide to Algorithmic Strategies and Trading Systems. Wiley, Hoboken (NJ), second edition, 2013.

[Bat03] A. Battauz. Quadratic hedging for asset derivatives with discrete stochastic dividends. Insur. Math. Econ., 32(2):229-243, 2003.

[BHR14] J. Brogaard, T. Hendershott, and R. Riordan. High-frequency trading and price discovery. Rev. Financ. Stud., 27(8):2267-2306, 2014.

[DJ05] A. Dubinsky and M. Johannes. Earnings announcements and equity options. Columbia University, working paper, 2005.

[DS98] F. Delbaen and W. Schachermayer. The fundamental theorem of asset pricing for unbounded stochastic processes. Math. Ann., 312(2):215-250, 1998.

[Eva11] K.P. Evans. Intraday jumps and US macroeconomic news announcements. J. Bank. Financ., 35(10):2511$2527,2011$.

[Fam70] E.F. Fama. Efficient capital markets: A review of theory and empirical work. J. Financ., 25(2):383-417, 1970.

[Fam91] E.F. Fama. Efficient capital markets: II. J. Financ., 46(5):1575-1617, 1991.

[Fon15] C. Fontana. Weak and strong no-arbitrage conditions for continuous financial markets. Int. J. Theor. Appl. Finan., 18(1):1550005, 2015.

[Ges79] R. Geske. A note on an analytical valuation formula for unprotected American call options on stocks with known dividends. J. Financ. Econ., 7(4):375-380, 1979.

[GS16] F. Gehmlich and T. Schmidt. Dynamic defaultable term structure modeling beyond the intensity paradigm. Math. Financ., forthcoming, 2016.

[HJ88] D. Heath and R. Jarrow. Ex-dividend stock price behavior and arbitrage opportunities. J. Bus., 61(1):95108,1988 .

[HLS15] T. Hendershott, D. Livdan, and N. Schürhoff. Are institutions informed about news? J. Financ. Econ., $117(2): 249-287,2015$.

[JS03] J. Jacod and A.N. Shiryaev. Limit Theorems for Stochastic Processes. Springer, Berlin, second edition, 2003.

[KK07] I. Karatzas and C. Kardaras. The numéraire portfolio in semimartingale financial models. Finance Stoch., 11(4):447-493, 2007.

[KV91] O. Kim and R.E. Verrecchia. Market reaction to anticipated announcements. J. Financ. Econ., 30(2):273309, 1991.

[KW14] D.H. Kim and J.H. Wright. Jumps in bond yields at known times. Federal Reserve Board, Washington, discussion paper, 2014.

[Lee12] S.S. Lee. Jumps and information flow in financial markets. Rev. Financ. Stud., 25(2):439-479, 2012.

[Lew14] M. Lewis. Flash Boys: A Wall Street Revolt. Norton \& Company, New York - London, 2014.

[LM08] S.S. Lee and P.A. Mykland. Jumps in financial markets: a new nonparametric test and jump dynamics. Rev. Financ. Stud., 21(6):2535-2563, 2008.

[PH06] E. Platen and D. Heath. A Benchmark Approach to Quantitative Finance. Springer, Berlin - Heidelberg, 2006.

[Pia01] M. Piazzesi. An econometric model of the yield curve with macroeconomic jump effects. NBER working paper no 8246, 2001.

[Pia05] M. Piazzesi. Bond yields and the Federal Reserve. J. Polit. Econ., 113(2):311-344, 2005.

[Pro04] P. Protter. Stochastic Integration and Differential Equations. Springer, Berlin - Heidelberg, 2.1 edition, 2004. 
[Ran11] J.G. Rangel. Macroeconomic news, announcements, and stock market jump intensity dynamics. J. Bank. Financ., 35(5):1263-1276, 2011.

[Rol77] R. Roll. An analytic valuation formula for unprotected American call options on stocks with known dividends. J. Financ. Econ., 5(2):251-258, 1977.

[Wha81] R.E. Whaley. On the valuation of American call options on stocks with known dividends. J. Financ. Econ., 9(2):207-211, 1981.

(Claudio Fontana) Laboratoire de Probabilités et Modèles Aléatoires, Paris Diderot University, FRANCE

E-mail address: fontana@math.univ-paris-diderot.fr

(Markus Pelger) Management Science \& Engineering Department, Stanford University, Huang Engineering Center, Stanford, CA, United States

E-mail address: mpelger@stanford.edu

(Eckhard Platen) School of Mathematical and Physical Sciences and Finance Discipline Group, University of Technology Sydney, Broadway NSW 2007, Sydney, Australia, and Department of Actuarial SCIEnCES, University of CAPE TOWN.

E-mail address: Eckhard.Platen@uts.edu.au 